\title{
Fade statistics of $\mathcal{M}$-turbulent optical links
}

\author{
Antonio Jurado-Navas 1,2* (D), José María Garrido-Balsells², Miguel Castillo-Vázquez², \\ Antonio Puerta-Notario ${ }^{2}$, Idelfonso Tafur Monroy ${ }^{1}$ and Juan José Vegas Olmos ${ }^{1}$
}

\begin{abstract}
A new and generalized statistical model, called Málaga or simply $\mathcal{M}$ distribution, has been derived recently to characterize the irradiance fluctuations of an unbounded optical wavefront propagating through a turbulent medium under all irradiance fluctuation conditions. The aforementioned model extends and unifies in a simple analytical closed-form expression most of the proposed statistical models for free-space optical (FSO) communications widely employed until now in the scientific literature. Based on that $\mathcal{M}$ model, we have studied some important features associated to its fade statistics and expressed in terms of the expected number of fades per second. The derived expressions become relevant for many aspects in a FSO system, especially those ones related to determine the optimum threshold in a receiver based on a direct detection scheme employing a fixed detection threshold.
\end{abstract}

\section{Introduction}

In the last decades, free-space optical (FSO) communication systems are receiving considerable attention [1-8] especially thanks to their inherent potential transmission capacity, much higher than that offered by radio transmission technologies. In addition, their narrow beam widths and their inherent license-free operation allows FSO systems to become appropriate candidates for secure, high-data-rate, cost-effective, wide-bandwidth communications.

However, even in clear sky conditions, FSO links may experience fading of the received signal intensity - called scintillation - associated to the interaction of light with turbulent atmosphere [1]. That turbulent-induced scintillation is seen as a statistic process and can seriously impair the behavior of a FSO link in terms of bit error rate or burst error rate, for example.

To minimize its degrading effect, we can design different techniques both in the transmitter side and/or in the receiver side. To this end, the behavior of any FSO system based on intensity modulation can be deduced from the probability density function (PDF) of the irradiance.

\footnotetext{
*Correspondence: antnav@fotonik.dtu.dk

${ }^{1}$ Department of Photonics Engineering, Technical University of Denmark

(DTU), Ørsted Plads, Building 358, 2800 Kgs. Lyngby, Denmark

${ }^{2}$ Department of Communications Engineering, University of Málaga, Campus

de Teatinos s/n, 29071 Málaga, Spain
}

Particularly in this type of scenario, most widely accepted irradiance PDF models have led to the consideration of a conditional random process $[1,6-8]$. Among the efforts made to obtain the most realistic statistical model valid in all turbulent regimes, a new and generalized statistical model, called Málaga or simply $\mathcal{M}$ distribution, was recently derived and validated $[7,8]$ to characterize the irradiance fluctuations of an unbounded optical wavefront (plane and spherical waves) propagating through a turbulent medium under all irradiance fluctuation conditions in homogeneous, isotropic turbulence. This Málaga distribution unifies most of the irradiance statistical models for FSO communications proposed in literature in a closed-form expression. Its conditional random process is made by a Gamma and a compound of a Nakagami-m distribution and a Rayleigh random phasor.

In this paper, we perform a study focused on an atmospheric optical system affected by a Málaga turbulence. This PDF model will allow to predict the probability of fade associated to that system, i.e., the probability of having crossings below an established threshold level in the receiver side. Hence, after a brief introduction of the Málaga statistical model (Section 2) and about the system model considered through this work (Section 3), since the optical beam will fluctuate randomly following the latter model, the expected number of fades per second affecting a FSO system is studied in Section 4, showing some

\section{Springer Open}

(c) The Author(s). 2017 Open Access This article is distributed under the terms of the Creative Commons Attribution 4.0 International License (http://creativecommons.org/licenses/by/4.0/), which permits unrestricted use, distribution, and reproduction in any medium, provided you give appropriate credit to the original author(s) and the source, provide a link to the Creative Commons license, and indicate if changes were made. 
interesting features associated to the $\mathcal{M}$ distribution. In fact, the Málaga PDF is shown to model any turbulent condition, covering the gaps in the, until now, existing distributions. This fact is clearly shown when analyzing that last parameter, showing that a system can be affected by a larger or smaller number of fades although the normalized irradiance variance will be maintained at a same constant level. Other widely extended distributions as, for instance, the lognormal and the gamma-gamma, will be unable to explain this aspect.

\section{Málaga statistical model for the turbulence}

Málaga distribution is based on a new small-scale propagation scheme including a new scattering component for the observed field, $U_{S}^{C}$ coupled to the line-of-sight (LOS) field term, $U_{L}$. The propagation scheme is illustrated in ([7] Fig. 1) (we recommend to read that work for a detailed description). Following notation of [7], the average power of the LOS term is represented by $\Omega$ while the average power of the total scatter components is denoted by $2 b_{0}=$ $E\left[\left|U_{S}^{C}\right|^{2}+\left|U_{S}^{G}\right|^{2}\right]$, with $U_{S}^{G}$ being the classic scattering field independent of the LOS contribution. Accordingly, the average power of every single scatter component is given by $E\left[\left|U_{S}^{C}\right|^{2}\right]=\rho 2 b_{0}$ and $E\left[\left|U_{S}^{G}\right|^{2}\right]=(1-\rho)$ $2 b_{0}$, for the coupled-to-LOS scattering term and for the classic scattering component received by off-axis eddies, respectively. The parameter $\rho$ shows the amount of scattering power coupled to the LOS component, ranging from 0 to 1 .

Then, the $\mathcal{M}$ PDF of the received irradiance $I$ is represented by:

$$
\begin{aligned}
& f_{I}(I)=A \sum_{k=1}^{\beta} a_{k} I^{\frac{\alpha+k}{2}}-1 K_{\alpha-k}\left(2 \sqrt{\frac{\alpha \beta I}{\xi_{g} \beta+\Omega^{\prime}}}\right), \\
& \left\{\begin{array}{l}
A \triangleq \frac{2 \alpha^{\frac{\alpha}{2}}}{\xi_{g}^{1+\frac{\alpha}{2}} \Gamma(\alpha)}\left(\frac{\xi_{g} \beta}{\xi_{g} \beta+\Omega^{\prime}}\right)^{\beta+\frac{\alpha}{2}} ; \\
a_{k} \triangleq\left(\begin{array}{c}
\beta-1 \\
k-1
\end{array}\right) \frac{\left(\xi_{g} \beta+\Omega^{\prime}\right)^{1-\frac{k}{2}}}{(k-1) !}\left(\frac{\Omega^{\prime}}{\xi_{g}}\right)^{k-1}\left(\frac{\alpha}{\beta}\right)^{\frac{k}{2}} .
\end{array}\right.
\end{aligned}
$$

In Eq. (1), $\beta \in \mathbb{N}$ is the shape parameter of the Nakagami distribution representing the amount of fading factor, with $\Omega^{\prime}=\Omega+\rho 2 b_{0}$ representing the average power from the coherent contributions, whereas $K_{v}(\cdot)$ is the modified Bessel function of the second kind and order $\nu$. Finally, $\alpha$ is a positive parameter related to the effective number of large-scale cells of the scattering process [6], and arisen from the gamma approximation to a lognormal distribution. In the interest of clarity, the algebraic manipulation to prove this result can be consulted in ([7]App. A).
A generalized PDF expression was also obtained in $[7,8]$ when $\beta \in \mathbb{R}$, though the inherent degree of freedom associated to the proposed distribution allows to model almost any behavior with the case of $\beta$ being a natural number.

\section{System model}

Consider an intensity modulation/direct detection (IM/DD) link using on-off keying (OOK). In these type of systems, and in absence of turbulence, the electrical current signal induced in the receiver by the action of the received optical wave can be written as:

$$
i=i_{S}+i_{N}
$$

after the integration of the received photocurrent for an interval $T_{0} \leq T_{b}$, with $T_{b}$ being the bit interval of the OOK system [9]. In Eq. (2), $i_{N}$ represents the shot noise caused by ambient light much stronger than the desired signal and/or by thermal noise in the electronics following the photodetector. Since it is considered as statistically independent to the desired signal, $i_{S}$, then it is supposed to be modeled as a zero-mean additive white Gaussian noise (AWGN) described by the following PDF:

$$
f_{n}(i)=\frac{1}{\sqrt{2 \pi \sigma_{N}}} \exp \left(-\frac{i^{2}}{2 \sigma_{N}^{2}}\right),
$$

with $\sigma_{N}^{2}$ representing the variance of the noise. Since an OOK modulation scheme is used, then the instantaneous transmitted power is either 0 or $2 P_{t}$, with $P_{t}$ being the average transmitted optical power. In this respect, $i_{S}=2 R P_{t}$ denotes the electrical current associated to the received signal light, with $R$ being the responsivity whereas $P_{t}$ is the aforementioned average of transmitted optical power. Accordingly, the total electrical current signal induced in the receiver, $i=i_{S}+i_{N}$, is also governed by the following non-zero mean Gaussian PDF:

$$
f_{s+n}(i)=\frac{1}{\sqrt{2 \pi \sigma_{N}}} \exp \left[-\frac{\left(i-i_{S}\right)^{2}}{2 \sigma_{N}^{2}}\right] .
$$

In the presence of atmospheric turbulence, the instantaneous transmitted optical power, $2 P_{t}$, suffers from a fluctuation in signal intensity. Hence, $i_{S}$ will suffer that aforementioned fluctuation too. From (2) it is straightforward to see that we have considered a receiving aperture smaller than the correlation length of the irradiance fluctuations, i.e., the aperture behaves essentially as a point detector [1] and ignores intersymbol interference, then the receiver detects signal light only when an 'on' state is transmitted. Assuming a point detector implies that the dark current can be neglected in the noise considerations. Indeed, and as indicated in [10], the dark current is reduced by cooling the detector or by reducing the 
physical size of the detector. Thus, if we consider a point detector, then dark current can be definitively neglected.

\section{Expected number of fades}

In FSO links, fades are associated with fluctuations in pressure of the atmosphere also induces in refractive index irregularities. Thus, the introduction of the atmosphere between source and receiver, and its inherent random refractive index variations, can lead to power losses at the receiver and eventually it produces spatial and temporal fluctuations in the received irradiance, i.e., turbulenceinduced signal power fading [1]. Such fluctuations can produce an increase in the link error probability limiting the performance of communication systems. In this particular scenario, the turbulence-induced fading is called scintillation. Since the scintillation sequence represents the effect of the intensity fluctuations on the transmitted signal, its adverse effect is notorious when an intensity modulated and direct detection (IM/DD) system is employed, especially when the detection threshold is fixed to a concrete value. The received signal will vary in intensity, and a number of undesired crossings to this threshold detection is recorded. The higher the intensity of the turbulence, the deeper the fades involved in the process and the larger the number of undesired crossings, increasing each undesired crossing the number of wrongly detected bits. Then, the bit error rate (BER) associated with these systems is directly affected.

In this section, we derive the analytical expression to obtain the number of non-desired crossings below a given threshold by the output current per unit time, represented by $<n\left(i_{u}\right)>$, under $\mathcal{M}$ turbulence. For a stationary process and for a high signal-to-noise ratio conditions at the detector, it was demonstrated $[11,12]$ that this $<n\left(i_{u}\right)>$ can be defined by:

$$
<n\left(i_{u}\right)>=0.5 \int_{-\infty}^{\infty}\left|i^{\prime}\right| f_{i, i^{\prime}}\left(i, i^{\prime}\right) \mathrm{d} i^{\prime},
$$

where $i^{\prime}=\frac{\mathrm{d} i}{\mathrm{~d} t}$, with $f_{i, i^{\prime}}\left(i, i^{\prime}\right)$ representing the joint PDF of the received current $i$ affected by the turbulent process and its time derivative $i^{\prime}$. Furthermore, the noise contribution to the expected number of fades has been neglected in Eq. (5).

As indicated in $[1,11,12]$, the time derivative of a random process and the process itself are uncorrelated, but not necessarily independent. Nevertheless, we can assume the assumption made in [1] for the gamma-gamma in which it was discussed that, although the conditional PDF of $i^{\prime}$ is not known, however, based on physical grounds, it can be approximated to a zero-mean Gaussian PDF. Supporting that statement, and as detailed in [13], the Málaga distribution tends to a zero-mean Gaussian PDF if $\alpha \rightarrow 1$, $\beta \rightarrow 1$, and $m_{k}=1$, with $m_{k}$ representing the probability that certain portion of the transmitted optical power travels through the $k$-th optical path, for which the probability of the optical power to be coupled to the line-of-sight component is established by an additional factor inherent to $m_{k}$, as shown in [14].

Thus, the joint PDF, $f_{i, i^{\prime}}\left(i, i^{\prime}\right)$, could be expressed as:

$$
f_{i, i^{\prime}}\left(i, i^{\prime}\right)=f_{i}(i) f_{i^{\prime}}\left(i^{\prime} \mid i\right),
$$

and based on the mentioned previous assumption, the PDF of $i^{\prime}$ can be approximated as a Gaussian one. Consequently, from [1],

$$
f_{i^{\prime}}\left(i^{\prime}\right)=\frac{1}{\sqrt{2 \pi b}} \exp \left(-\frac{i^{\prime 2}}{2 b}\right)
$$

where $b=-<I>B_{I}^{\prime \prime}(0) / 4$ represents the standard deviation of the time derivative of a Gaussian random process, with $B_{I}^{\prime \prime}(x)$ being the second derivative of the covariance function of received irradiance whereas $<>$ represents the ensemble average operator. Finally, $f_{i}(i)$ is the irradiance PDF written in (1).

After rearranging terms, the resolution of (5) is reduced to solve the following integrate:

$$
\int_{-\infty}^{\infty}|t| \exp \left(-t^{2}\right) \mathrm{d} t=1
$$

Hence, we can deduce that:

$$
\begin{aligned}
<n\left(i_{u}\right)>= & \frac{\sqrt{2 b}}{\sqrt{\pi}} A \sum_{k=1}^{\beta} a_{k}\left(\frac{i_{u}}{<I>}\right)^{\frac{\alpha+k-1}{2}} \\
& \times K_{\alpha-k}\left(2 \sqrt{\frac{\alpha \beta i_{u}}{\left(\xi_{g} \beta+\Omega^{\prime}\right)<I>}}\right)
\end{aligned}
$$

Now we can take into account the concept of quasifrequency, $v_{0}$, defined as the frequency of fades when $i_{u}=i_{S}$ and, consequently, seen as a measure of the effective bandwidth of the current signal in the receiver. This magnitude expressed in terms of $b$, as:

$$
\nu_{0}=\frac{1}{2 \pi} \sqrt{-\frac{B_{I}^{\prime \prime}(0)}{B_{I}(0)}}=\frac{1}{\pi \sigma_{I}} \sqrt{\frac{b}{<I>}} \quad[\mathrm{Hz}]
$$

In [15], a Gaussian model for the temporal covariance function of the irradiance, $B_{I}(\cdot)$ was presented in the form:

$$
B_{I}(\tau)=\sigma_{I}^{2} \exp \left(-\frac{\tau^{2}}{\tau_{0}^{2}}\right)
$$

where $\tau_{0}=\frac{\sqrt{\lambda L}}{u_{\perp}}$ is the turbulence correlation time, $\lambda$ is the optical wavelength, and $L$ is the propagation path length satisfying that $l_{0}<L<L_{0}$, with $l_{0}$ and $L_{0}$ being the inner scale and the outer scale of the turbulence. Finally, $u_{\perp}$ represents average wind speed transverse to the propagation direction. As an additional explanation provided in [16], that $\tau_{0}$ parameter denotes the time required for 
an inhomogeneity moving with velocity $u_{\perp}$ to cover a distance $\sqrt{\lambda L}$, with this last distance representing the fading correlation length [9]. In (11), it was assumed that the Taylor's hypothesis of frozen turbulence $[17,18]$ is satisfied. Under this hypothesis, eddies do not change significantly during the time they drift through the beam, i.e., the collection of atmospheric eddies formed in the atmosphere following, for instance, the Kolmogorov cascade theory [1], will remain frozen in relation to one another, while the entire collection is transported as a whole along some direction by the wind. Furthermore, based on the Taylor frozen turbulence hypothesis, spatial and temporal statistics are completely related by knowledge of the mean wind speed transverse to the direction of propagation, $u_{\perp}$. Now, calculating the second derivative to (11),

$$
B_{I}^{\prime \prime}(\tau)=-\frac{2 \sigma_{I}^{2}}{\tau_{0}^{2}} \exp \left(-\frac{\tau^{2}}{\tau_{0}^{2}}\right)\left[1+\frac{2 \tau^{2}}{\tau_{0}^{2}}\right],
$$

and substituting its expression in addition to the expression of the temporal covariance function into (10), the value of $b$ can be obtained:

$$
b=\left(\nu_{0} \pi \sigma_{I}\right)^{2}<I>\text {. }
$$

On another note, if we evaluate (12) in $\tau=0$, then we can obtain

$$
B_{I}^{\prime \prime}(0)=-\frac{2 \sigma_{I}^{2}}{\tau_{0}^{2}} .
$$

Then, substituting (14) in (12) then we can obtain a relationship between the quasi-frequency and the turbulence correlation time:

$$
\nu_{0}=\frac{1}{\pi \tau_{0} \sqrt{2}}
$$

Hence, the expected number of fades below the threshold can be written as:

$$
\begin{aligned}
<n\left(i_{u}\right)>= & \frac{\sigma_{I} \sqrt{<I>}}{\tau_{0} \sqrt{\pi}} A \sum_{k=1}^{\beta} a_{k}\left(\frac{i_{u}}{<I>}\right)^{\frac{\alpha+k-1}{2}} \\
& \times K_{\alpha-k}\left(2 \sqrt{\frac{\alpha \beta i_{u}}{\left(\xi_{g} \beta+\Omega^{\prime}\right)<I>}}\right)
\end{aligned}
$$

Figure 1 displays the expected number of fades associated to a Málaga turbulence as a function of fade threshold parameter, $F_{T}=10 \cdot \log _{10}\left(\frac{<I>}{i_{u}}\right)$. In that Fig. 1, we have assumed a propagation path length of $200 \mathrm{~m}$, an optical wavelength of $1550 \mathrm{~nm}$, and a wind speed transverse to the propagation direction of $u_{\perp}=10 \mathrm{~m} / \mathrm{s}$. Additionally, we have considered the intensities of turbulence and turbulent channel parameters included in Table 1, offering a wide range of turbulence regimes, from very weak to strong turbulence conditions. We must indicate that for the case of very weak turbulence $\left(\sigma_{I}^{2}=0.061\right)$, we have not registered undesired threshold crossings. For the sake of clarity, it is embed in the same figure but with the Y-axis in linear scale. Some numerical results obtained by Monte Carlo simulation are provided. For that numerical simulation, it is assumed that a same scintillation coefficient is maintained during the interval of time defined by the atmospheric time of coherence, $\tau_{0}$.

After observing Fig. 1, and recalling that it was obtained for a high signal-to-noise ratio conditions at the detector, we can verify that the optimum threshold to minimize the expected number of undesired fadings is achieved when $F_{T} \rightarrow 0$, i.e., when the threshold is placed near the position associated to a binary logic ' 0 '.

Conversely, we can obtain the critical threshold value associated with each turbulence regime so that the maximum number of threshold crossings occurs. These critical thresholds represent the worst case of operation for each turbulence regime. In this respect, Table 2 shows such values considering that the transmitted peak power per pulse was established to $i_{S}=0.6 \mu \mathrm{W}$. There, the desired signal is written as $i_{S}=\mathbb{K} i_{u}$, with $i_{u}$ denoting the detection threshold, and with $\mathbb{K}$ being a positive parameter.

As we can observe, the weaker the turbulence strength is, the closer the critical threshold value is with respect to the mean received electrical current signal induced in the receiver. On the contrary, when the turbulenceinduced fluctuation becomes stronger, a displacement of the critical threshold towards the value representing the transmitted binary logic ' 0 ' is observed. This is the expected behavior, especially considering the initial hypothesis involving Eq. (5) that the AWGN noise contribution to the expected number of fades affecting the system can be neglected, i.e., the signal-to-noise ratio at the detector stage is sufficiently high. In this regard, the weaker the turbulence, the narrower the PDF associated to the scintillation process and the more concentrated around its mean value. Precisely, a threshold established around that mean value will cause the maximum expected number of threshold crossings, as it is shown in Fig. 1 and Table 2. For that reason, and assuming a high SNR operation mode, to minimize the missed detection probability, $P_{r}(0 \mid 1)$, the optimal threshold must be placed out of this critical zone in the PDF.

Conversely, if the turbulence intensity becomes stronger, then its associated PDF becomes wider and the set of potential values for the received level of power corresponding to a binary logic ' 1 ' is increased. Therefore, the stronger the turbulence, the further from the ideal logic ' 1 ' mean value the critical threshold is situated, where that critical threshold represents, again, that one with which $P_{r}(0 \mid 1)$ is maximized. Figure 2 shows a scintillation sequence with a $\sigma_{I}^{2}=1.21$ and obtained with a $u_{\perp}=10 \mathrm{~m} / \mathrm{s}$. The amplitude is normalized to the transmitted power level associated with an 'on' state. It is also shown, following Table 2, the critical threshold 


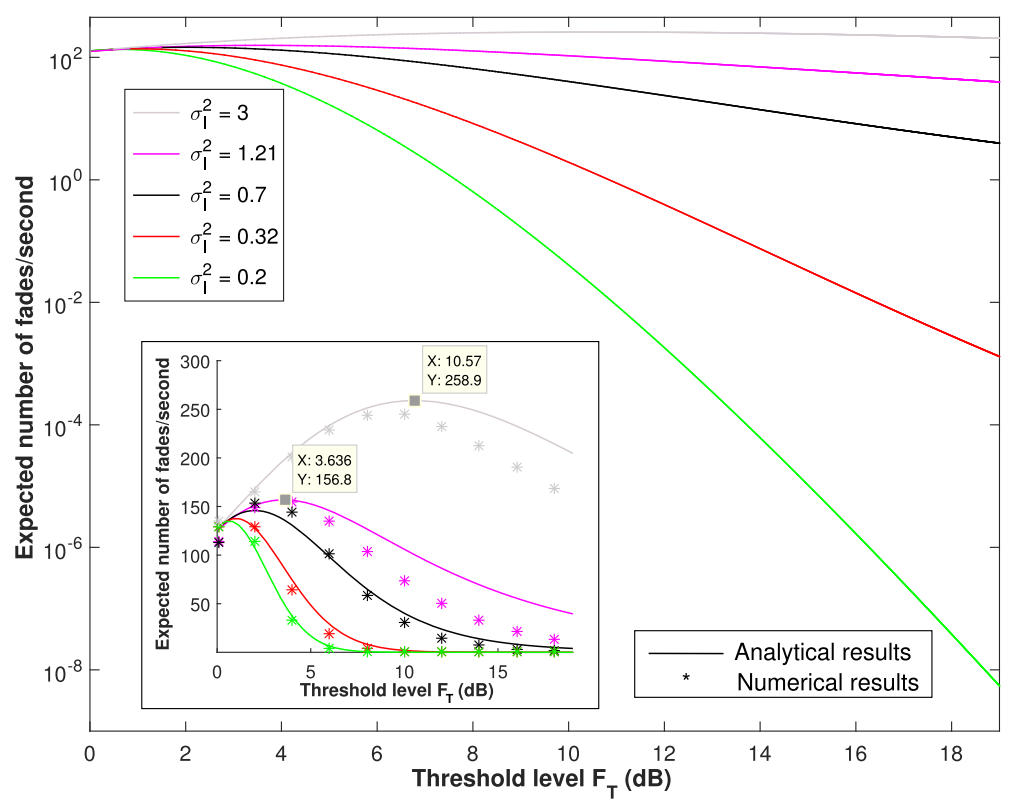

Fig. 1 Expected number of fades versus $F_{T}=10 \cdot \log _{10}\left(\frac{<l>}{i_{u}}\right)$ for a Málaga turbulence model. Turbulent channel parameters $(\alpha, \beta, \Omega, \rho)$ were taken from Table 1. Inset shows the same figure but with the $Y$-axis in linear scale

corresponding that intensity of turbulence: $i_{u}=\frac{1}{2.3121}$, i.e., $\mathbb{K}=2.3121$. As it can be observed, the larger number of undesired threshold crossings occurs in the value represented by that critical threshold.

On another note, Fig. 3 shows a comparative of the expected number of fades when the crosswind speed is reduced to $u_{\perp}=10 \mathrm{~m} / \mathrm{s}$ to $u_{\perp}=1 \mathrm{~m} / \mathrm{s}$. It is straightforward to check that a larger mean wind speed transverse to the light propagation direction induces a larger number of expected fades since the atmospheric correlation time, $\tau_{0}$ becomes smaller and thus, the mean width of each fading is being reduced when the crosswind speed is increased. Approximately, there are ten times more expected fades for $u_{\perp}=10 \mathrm{~m} / \mathrm{s}$ than for the case of $u_{\perp}=1 \mathrm{~m} / \mathrm{s}$. This feature is completely expected from Eq. (16), where an inverse relationship between the expected number of fades and the turbulence correlation time, $\tau_{0}$, was established. Consequently, there is a direct relationship between the expected number of fades wind speed transverse to the propagation direction, $u_{\perp}$, since $\tau_{0}=$ $\sqrt{\lambda L} / u_{\perp}$. As indicated before, for the case of very weak turbulence $\left(\sigma_{I}^{2}=0.061\right)$, we have not observed any undesired threshold crossing for both wind velocities.

Finally, we have evaluated the difference in behavior associated with the Málaga PDF for a same turbulence intensity. Figure 4 shows the expected number of fades per second for up to six different scintillation sequences following a $\mathcal{M}$ statistical model. The variance is fixed to $\sigma_{I}^{2}=1.21$ for all cases. We have evaluated five cases in which the average power of the LOS term is established to a same value $(\Omega=0.39)$, whereas the amount of scattering power coupled to the LOS component, $\rho$ varies from 0.12 to 0.82 . For the case of Fig. 4a, a direct relationship can be determined between $\rho$ and the expected number of
Table 1 Some illustrative scenarios following a Málaga statistical model and employed to obtain Fig. 1

\begin{tabular}{lllll}
\hline$\alpha$ & $\beta$ & $\Omega$ & $\rho$ & $\sigma_{I}^{2}$ \\
\hline 1 & 1 & 0.8333 & 0.82 & 3.00 \\
4 & 3 & 0.39 & 0.33 & 1.21 \\
8 & 2 & 0.87 & 0.890 & 0.70 \\
10 & 5 & 0.99 & 0.75 & 0.32 \\
11 & 10 & 0.9999 & 0.9999 & 0.20 \\
200 & 20 & 0.95 & 0.94 & 0.061 \\
\hline
\end{tabular}

Table 2 Worst-case threshold for OOK and Málaga atmospheric channel, with $u_{\perp}=10 \mathrm{~m} / \mathrm{s}$ and $i_{S}=0.6 \mu \mathrm{W}$

\begin{tabular}{llll}
\hline$\sigma_{l}^{2}$ & $i_{u}$ & $\mathbb{K}$ & $F_{T}$ \\
\hline 3.00 & $7.225 \cdot 10^{-8}$ & 11.3501 & 10.55 \\
1.21 & $3.5466 \cdot 10^{-7}$ & 2.3121 & 3.64 \\
$0.70 \cdot 10^{-1}$ & $5.0935 \cdot 10^{-7}$ & 1.6099 & 2.068 \\
$0.32 \cdot 10^{-2}$ & $6.5135 \cdot 10^{-7}$ & 1.2589 & 1.00 \\
$0.20 \cdot 10^{-2}$ & $7.0755 \cdot 10^{-7}$ & 1.1589 & .6405 \\
$0.061 \cdot 10^{-3}$ & No crossings & $\rightarrow 1$ & $\rightarrow 0$ \\
\hline
\end{tabular}




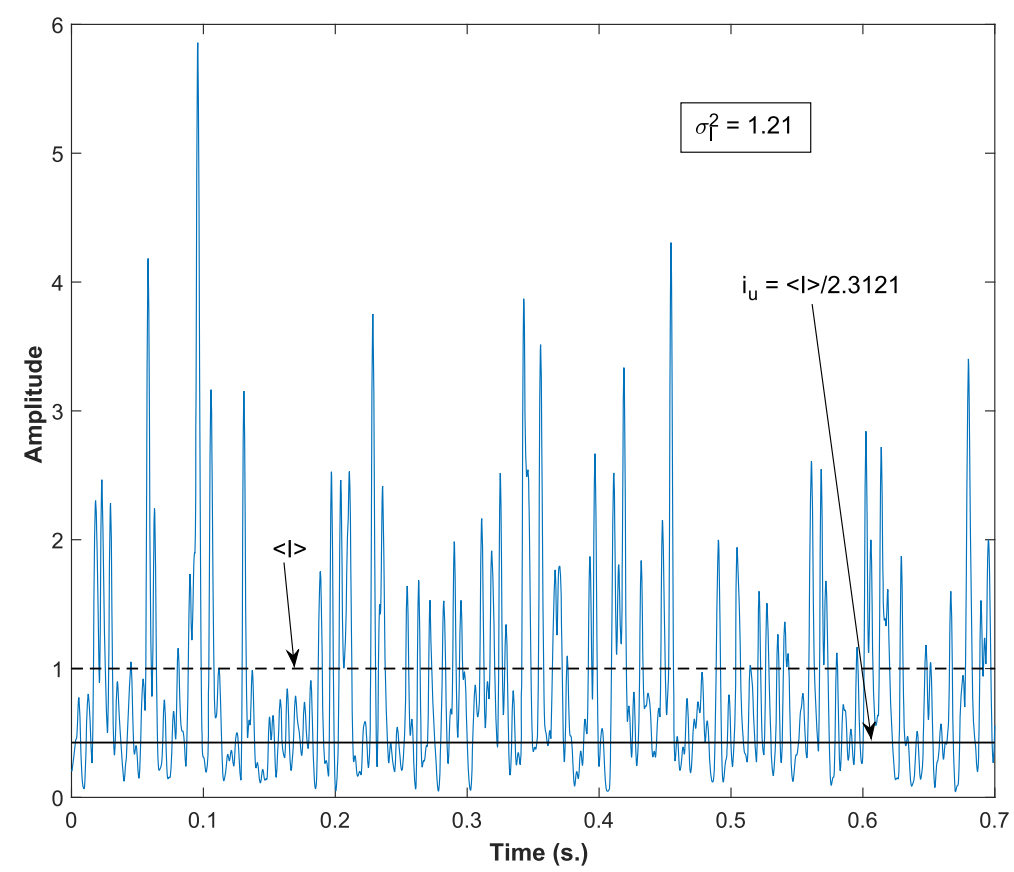

Fig. 2 Typical Málaga scintillation sequence with an ensemble average of 1 and a variance fixed to 1.21. The critical threshold is represented with a dash line and its value is coincident with the expected one shown in Table 2

fades per second. Hence, when there exists a higher contribution of the scattering component that travels coupled to the LOS field term, then the system is less affected by fading when $F_{T}$ is sufficiently high (for this particular scenario, $F_{T}>9 \mathrm{~dB}$ ). On the contrary, if the scattering power is mostly seen as a component independent to the lineof-sight one, then the number of threshold crossings will increase. These effects are more remarkable when $F_{T}$ is larger. For instance, for the case of $F_{T}=18 \mathrm{~dB}$, then the difference oscillates between 47.56 and 27.58 for the cases

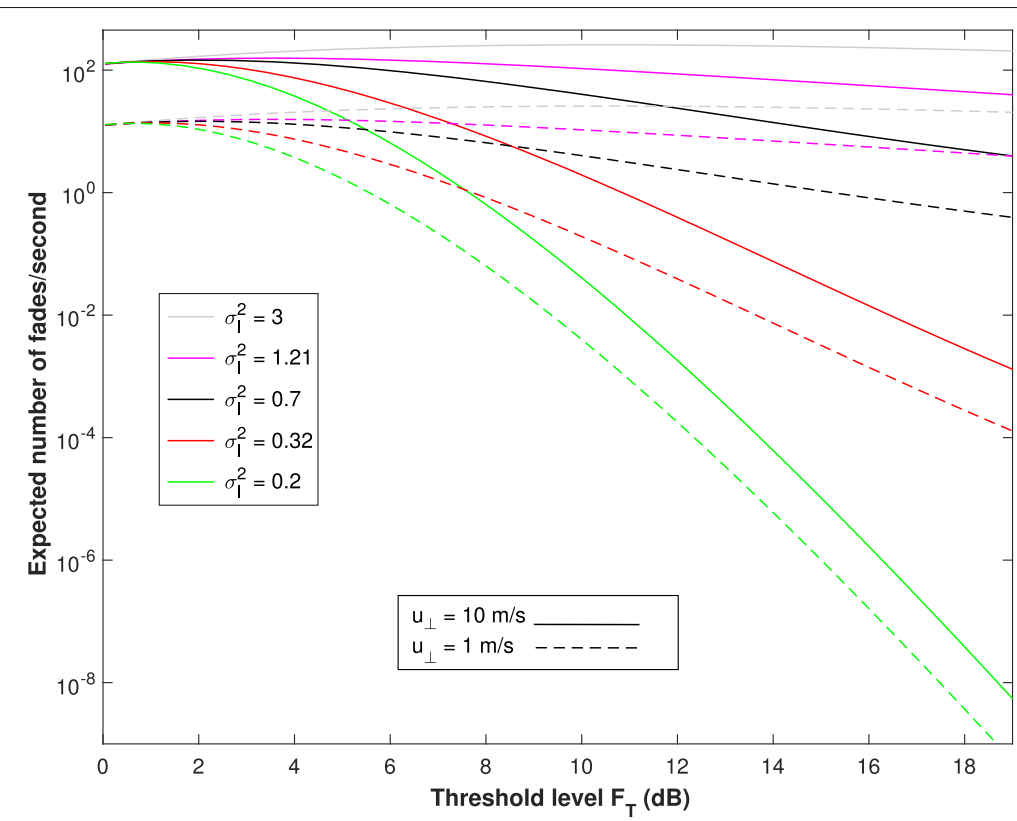

Fig. 3 Expected number of fades versus $F_{T}=10 \cdot \log _{10}\left(\frac{<>>}{i_{u}}\right)$ for a Málaga turbulence model for two different crosswind speeds: $u_{\perp}=1$ and $u_{\perp}=10 \mathrm{~m} / \mathrm{s}$. Turbulent channel parameters $(\alpha, \beta, \Omega, \rho)$ were taken from Table 1 


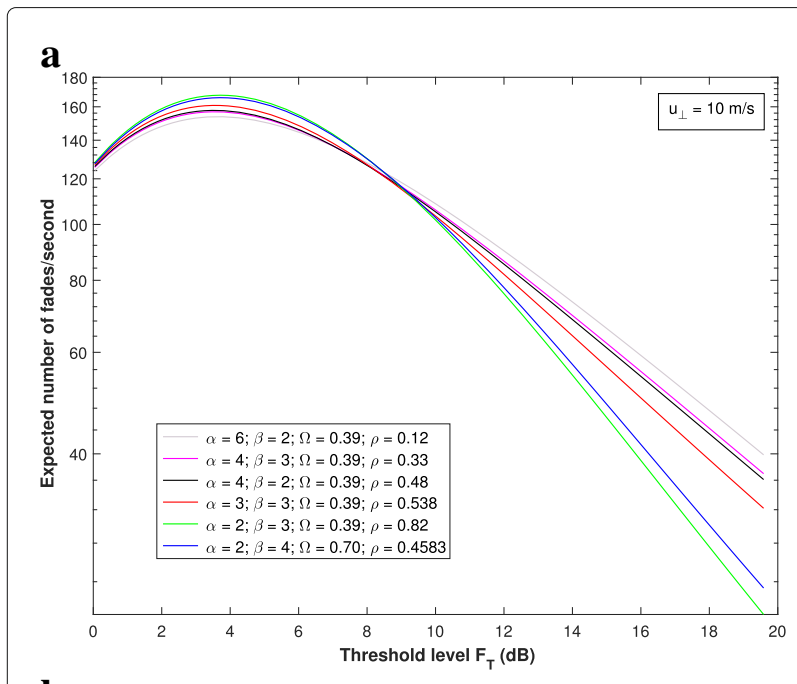

b

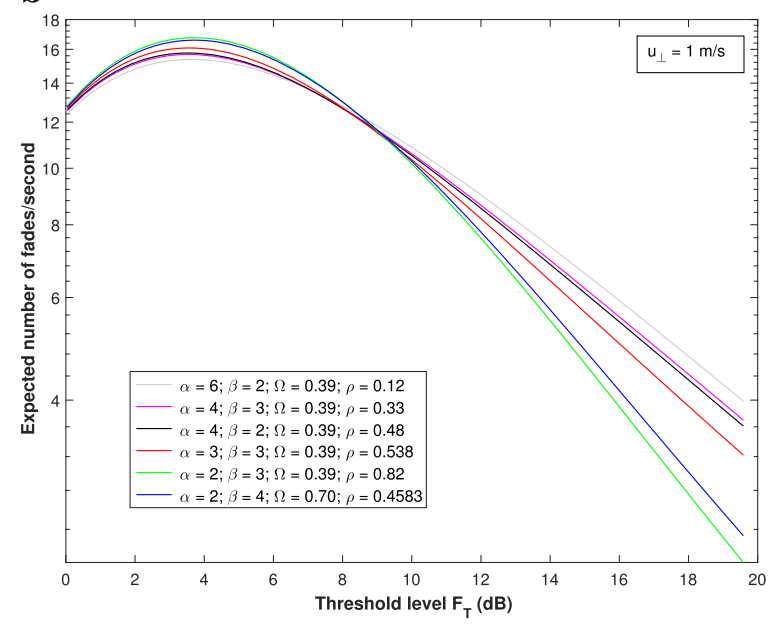

Fig. 4 Different behaviors of the Málaga PDF for a same intensity of turbulence $\sigma_{l}^{2}=1.21$ and a crosswind speed of $\mathbf{a} 10 \mathrm{~m} / \mathrm{s}$, and $\mathbf{b} 1 \mathrm{~m} / \mathrm{s}$

of $\rho=0.82$ and $\rho=0.12$, respectively. However, for the case of $F_{T}<9 \mathrm{~dB}$, the tendency is inverted. Thus, for the case of $F_{T}=3.64 \mathrm{~dB}$, the number of expected fades varies from 167.5 for the case of $\rho=0.82$; to 153.8 for the case of $\rho=0.12$.

This difference in behaviors is directly related to different temporal realizations for every Málaga scintillation sequence studied in Fig. 4. This effect is clearly seen when we represent the histograms. Accordingly, we have displayed in Fig. 5 several histograms corresponding to the significant cases of $\rho=0.12$ (Fig. 5a), $\rho=0.48$ (Fig. 5b), and $\rho=0.82$ (Fig. 5 c); the three of them with $\Omega=0.39$ whereas Fig. $5 \mathrm{~d}$ represents the case of $\rho=0.4583$ but with $\Omega=0.7$.

When the amount of power traveling through the line-of-sight propagation path is increased (the case of $\Omega=0.7$ ), the resulting curve is not similar to the analogous one for $\Omega=0.39$ and similar value of $\rho$; on the contrary, its behavior is more related to the case of $\Omega=$ 0.39 and $\rho=0.82$. In fact, for this last case, the total amount of power (from LOS contribution and from the scattering-coupled-to-LOS component) is closer (0.95) to the curve of $\Omega=0.7$, with that fact being a plausible explanation of this effect.

In addition, we can confirm another meaningful feature affecting all the curves (including the blue one with a higher LOS power): the critical threshold detection, the point where a higher number of expected fades is registered, is placed at the same position, that is exactly the value calculated in Table 2: $F_{T}=3.64$, or, in other words, $i_{u}=<I>/ 2.3121$. Hence, this critical threshold does not depend on the set of parameters from the Málaga PDF but it depends on solely its variance. Hence, for a concrete turbulence regime characterized by a particular $\sigma_{I}^{2}$, no matter how much power will be traveling through the LOS path, and no matter which amount of scattering power is coupled to the LOS term, the critical threshold detection is always maintained at the same value.

Eventually, similar conclusions can be derived for Fig. 4b when $u_{\perp}$ was reduced to $1 \mathrm{~m} / \mathrm{s}$.

\section{Conclusions}

In this paper, we have presented new different features involving the $\mathcal{M}$ generalized statistical model. First, we have analyzed the performance of an IM/DD optical system employing an OOK scheme when the detection threshold is fixed. In that case, the system is unable to adapt itself to the varying conditions of the channel, and a limit in terms of error floor is obtained. In particular, the analytical closed-form expression for that error rate was derived in this paper for different turbulent regimes showing that is precisely its intensity, in terms of $\sigma_{I}^{2}$, the parameter that determines the value of the error floor. Hence, the less intensity the turbulent process is, the lower the error floor is reached in terms of bit error rate.

Next, for any optical system employing a threshold detection, we have calculated the expected number of fades affecting the receiver considering, as in the whole paper, the $\mathcal{M}$ atmospheric model. Obtained equations were related to the value of the mean wind speed transverse to the light propagation direction. We have demonstrated that a higher wind speed induces a larger number of fades per second in the system. Certainly, and providing that the Taylor's hypothesis of frozen turbulence $[17,18]$ is satisfied, that increase in the mean wind speed transverse to the propagation path will not affect the bit error rate since this one is a first-order statistic; but a figure of merit expressed in terms of burst error rate will be completely affected.

On another note, we have calculated the critical detection threshold in the system, seen it as the threshold value so that the missed detection probability is maximized 
a

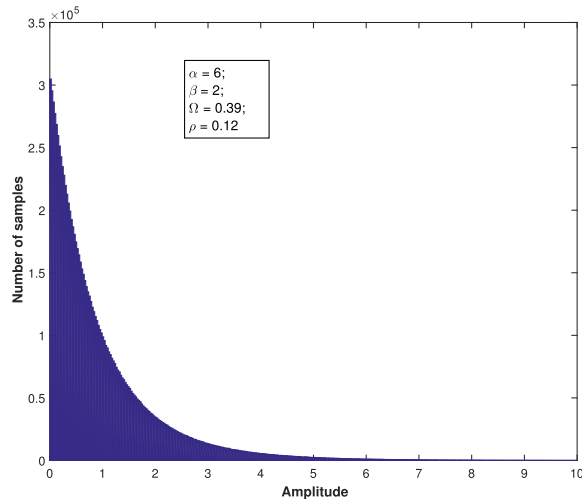

b

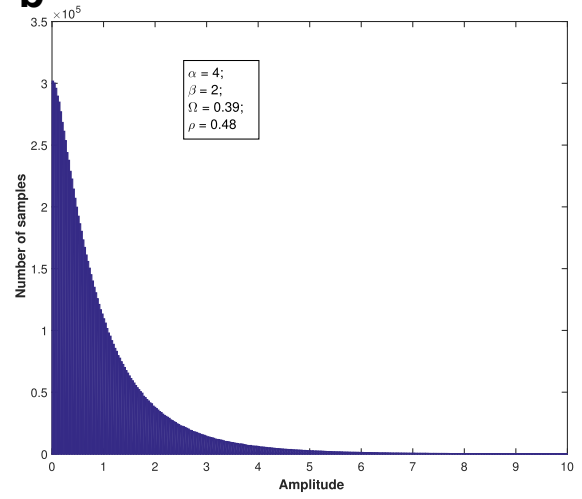

c

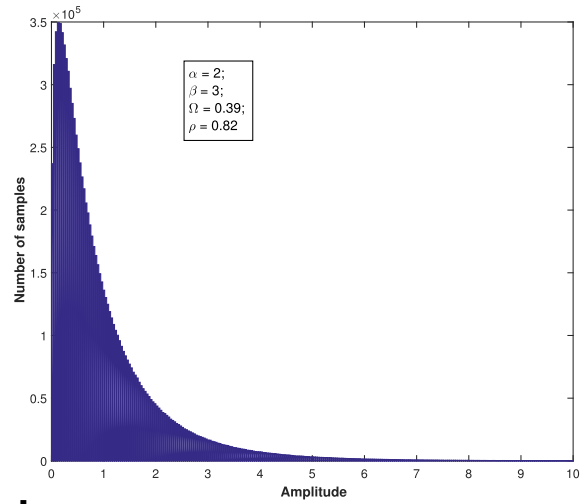

d

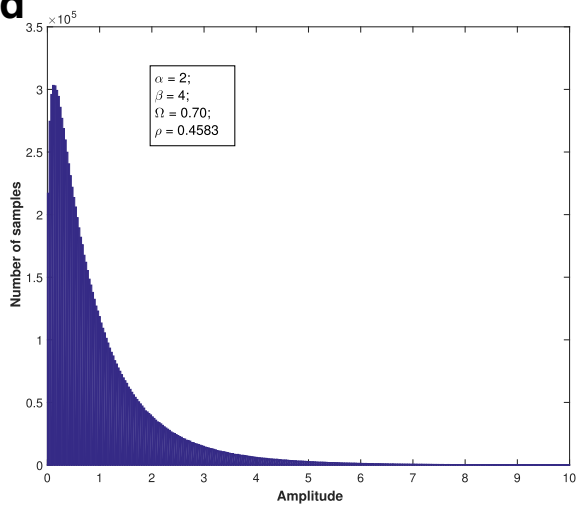

Fig. 5 Different histograms of a Málaga PDF. In subfigures a-c, the LOS power was established to 0.39 , and $\rho$ was taking, respectively, the following values: $0.12,0.48$, and 0.82. In subfigure $\mathbf{d}$, the LOS power was increased to 0.7 . In all cases, $\sigma_{l}^{2}=1.21$

because it suffers the largest number of undesired crossings. The weaker the turbulence variance is registered, the closer to the ideal mean 'on'-level the threshold is placed.

Finally, we have displayed a feature associated to the Málaga PDF, showing its robustness and universality. For a same value of $\sigma_{I}^{2}$, we can obtain different behaviors both depending on the amount of scattering power coupled to the line of sight and depending on the amount of nonscattering power traveling through the LOS path. Hence, the expected number of fades per second shows some differences, more remarkable when $F_{T}$ is becoming larger. Nevertheless, the critical threshold is always obtained at the same point, being solely affected by the intensity of turbulence. This fact can facilitate the design of new optical systems because regardless of the complexity inherent to the turbulence process, however, the rough knowledge of its intensity is sufficient to avoid the threshold area affected by a larger number of fades per second.

\section{Funding}

This work was supported by the Andalucía Talent Hub Program launched by the Andalusian Knowledge Agency, co-funded by the European Union's Seventh Framework Program, Marie Skłodowska-Curie actions (COFUND Grant Agreement no 291780).

\section{Authors' contributions}

The main idea of this paper was arisen from the discussion among AJN, JMGB, $M C V$, and APN. AJN developed the system model. ITM and JJVO provided a new focus for this work, and all authors contributed to the interpretation of the results and writing of the manuscript. All authors read and approved the final manuscript.

\section{Competing interests}

The authors declare that they have no competing interests.

\section{Publisher's Note}

Springer Nature remains neutral with regard to jurisdictional claims in published maps and institutional affiliations.

Received: 19 March 2016 Accepted: 2 June 2017

Published online: 21 June 2017

\section{References}

1. LC Andrews, RL Phillips, CY Hopen, Laser beam scintillation through random media. (SPIE, Bellingham, 2005)

2. K Kazaura, K Wakamori, M Matsumoto, T Higashino, K Tsukamoto, S Komaki, RoFSO: a universal platform for convergence of fiber and free-space optical communication networks. IEEE Comm. Mag. 48(2), 130-137 (2010)

3. A Jurado-Navas, JM Garrido-Balsells, M Castillo-Vázquez, A Puerta-Notario, An efficient rate-adaptive transmission technique using shortened pulses for atmospheric optical communications. Opt. Express. 18(16), 6-17363 (2010) 
4. JJ Vegas Olmos, X Pang, A Lebedev, M Sales Llopis, I Tafur Monroy, Wireless and wireline service convergence in next generation optical access networks - the FP7 WISCON project. IEICE Trans. Commun. E97-B(8), 1537-1546 (2014)

5. A Jurado-Navas, A Tatarczak, X Lu, JJ Vegas Olmos, JM Garrido-Balsells, I Tafur Monroy, 850-nm hybrid fiber/free-space optical communications using orbital angular momentum modes. Opt. Express. 23(26), 33721-33732 (2015)

6. MA Al-Habash, LC Adrews, RL Phillips, Mathematical model for the irradiance probability density function of a laser beam propagating through turbulent media. Opt. Eng. 40(8), 1554-1562 (2001)

7. A Jurado-Navas, JM Garrido-Balsells, JF Paris, A Puerta-Notario, Numerical simulations of physical and engineering processes. (In-Tech, Rijeka, 2011), pp. 181-206

8. A Jurado-Navas, JM Garrido-Balsells, JF Paris, M Castillo-Vázquez, A Puerta-Notario, General analytical expressions for the bit error rate of atmospheric optical communicatino systems: erratum. Opt. Lett. 39(20), 5896-5896 (2014)

9. X Zhu, JM Kahn, Free space optical communication through atmospheric turbulence channels. IEEE Trans. Commun. 50(8), 1293-1300 (2002)

10. H Kaushal, VK Jain, S Kar, Free space optical communication. (Springer, New Delhi, 2017)

11. SO Rice, The mathematical analysis of random noise. J. Bell Sys. Tech. 23(3), 282-332 (1944)

12. SO Rice, The mathematical analysis of random noise. J. Bell Sys. Tech. 24(1), 46-156 (1945)

13. A Jurado-Navas, JM Garrido-Balsells, JF Paris, M Castillo-Vázquez, A Puerta-Notario, in Proceedings of 2012 International Workshop on Optical Wireless Communications (IWOW). Further insights on Málaga distribution for atmospheric optical communications, (Pisa, 2012)

14. JM Garrido-Balsells, A Jurado-Navas, JF Paris, M Castillo-Vázquez, A Puerta-Notario, Novel formulation of the M model through the Generalized-K distribution for atmospheric optical channels. Optics Express. 23(5), 6345-6358 (2015)

15. A Jurado-Navas, A García-Zambrana, A Puerta-Notario, Efficient lognormal channel model for turbulent FSO communications. IET Elect. Lett. 43(3), 178-179 (2007)

16. A Jurado-Navas, A Puerta-Notario, Generation of correlated scintillations on atmospheric optical communications. J. Opt. Commun. Netw. 1(5), 452-462 (2009)

17. VI Tatarskii, The effects of the turbulent atmosphere on wave propagation. (Israel Program for Scientific Translations, Jerusalem, 1971)

18. GI Taylor, The spectrum of turbulence. Proc. R. Soc. London. Series A Math. Physi. Sci. 164(919), 476-490 (1938)

\section{Submit your manuscript to a SpringerOpen ${ }^{\circ}$ journal and benefit from:}

- Convenient online submission

- Rigorous peer review

- Open access: articles freely available online

- High visibility within the field

- Retaining the copyright to your article

Submit your next manuscript at $\gg$ springeropen.com 